\title{
Association study of a brain-derived neurotrophic- factor polymorphism and short-term antidepressant response in major depressive disorders
}

\author{
Eugene Lin ${ }^{1,7}$ \\ Po See Chen ${ }^{2,6,7}$ \\ Lung-Cheng Huang 3,4 \\ Sen-Yen $\mathrm{Hsu}^{5}$ \\ 'Vita Genomics, Inc., Wugu Shiang, \\ Taipei, Taiwan; ${ }^{2}$ Department \\ of Psychiatry, Hospital and College \\ of Medicine, National Cheng \\ Kung University, Tainan, Taiwan; \\ ${ }^{3}$ Department of Psychiatry, National \\ Taiwan University Hospital Yun-Lin \\ Branch, Taiwan; ${ }^{4}$ Graduate Institute \\ of Medicine, Kaohsiung Medical \\ University, Kaohsiung, Taiwan; \\ ${ }^{5}$ Department of Psychiatry, Chi Mei \\ Medical Center, Liouying, Tainan, \\ Taiwan; ${ }^{6}$ Department of Psychiatry, \\ National Cheng Kung University \\ Hospital, Dou-liou Branch, Yunlin, \\ Taiwan; ${ }^{7}$ These authors contributed \\ equally to this work
}

\begin{abstract}
Major depressive disorder (MDD) is one of the most common mental disorders worldwide. Single nucleotide polymorphisms (SNPs) can be used in clinical association studies to determine the contribution of genes to drug efficacy. A common SNP in the brain-derived neurotrophic factor (BDNF) gene, a methionine (Met) substitution for valine (Val) at codon 66 (Val66Met), is a candidate SNP for influencing antidepressant treatment outcome. In this study, our goal was to determine the relationship between the Val66Met polymorphism in the BDNF gene and the rapid antidepressant response to venlafaxine in a Taiwanese population with MDD. Overall, the BDNF Val66Met polymorphism was found not to be associated with short-term venlafaxine treatment outcome. However, the BDNF Val66Met polymorphism showed a trend to be associated with rapid venlafaxine treatment response in female patients. Future research with independent replication in large sample sizes is needed to confirm the role of the BDNF Val66Met polymorphism identified in this study.
\end{abstract}

Keywords: antidepressant response, brain-derived neurotrophic factor, major depressive disorder, serotonin and norepinephrine reuptake inhibitor, single nucleotide polymorphisms

\section{Introduction}

Major depressive disorder (MDD) is one of the most common mental disorder worldwide (Kessler et al 2003). However, no single antidepressant has ever been shown to be more effective than any other in lifting depression (Lin et al 2006a). The effectiveness of medical treatments in individual MDD patients is unknown, and therefore doctors can only prescribe antidepressants based on a trial and error approach. (Lin et al 2006a). Single nucleotide polymorphisms (SNPs) can be used in clinical association studies to determine the contribution of genes to drug efficacy, as evidence is accumulating to suggest that the drug efficacy of antidepressants results from the combined effects of a number of genetic variants such as SNPs (Serretti and Artioli 2004; Kirchheiner et al 2004; Lin et al 2006a; Lin and Chen 2008).

A common SNP in the brain-derived neurotrophic factor (BDNF) gene, a methionine (Met) substitution for valine (Val) at codon 66 (Val66Met), is associated with alterations in brain anatomy and memory (Chen et al 2006). The BDNF gene encodes a protein of the nerve growth factor family that regulates synaptic plasticity and connectivity in the brain (Bath and Lee 2006). The relationship between BDNF and depression remains unclear and needs to be reassessed (Groves 2007). Recent studies indicated that the Val66Met polymorphism in the BNDF gene may play a key role in genetic predispositions to depressive disorders (Hwang et al 2006; Ribeiro et al 2007). In contrast, other studies have reported no association between this BDNF polymorphism and depression (Tsai et al 2003; Choi et al 2006). 
Based on various antidepressants, several studies in different populations support the implication of BDNF involvement in antidepressant treatment outcome (Tsai et al 2003; Choi et al 2006; Yoshida et al 2007). In a Korean population of MDD patients, Choi and colleagues (2006) found that the polymorphism Val66Met in the BNDF gene was associated with citalopram efficacy, with M-allele carriers responding better to citalopram treatment. Similarly, in a sample of Japanese patients with MDD, the BDNF Val66Met polymorphism was found to be associated with the antidepressant response to milnacipran and fluvoxamine (Yoshida et al 2007). Furthermore, it has been shown that heterozygous patients had a trend to improved 4-week-fluoxetine antidepressant response in comparison to homozygous carriers in another study of Taiwanese MDD patients (Tsai et al 2003).

On the contrary, the BNDF gene was genotyped and was not linked with citalopram treatment outcome in the Sequenced Treatment Alternatives for Depression (STAR*D) reports by McMahon and colleagues (2006) and Paddock and colleagues (2007). The STAR*D study is a large cohort of outpatients with MDD who were treated with the antidepressant citalopram (Rush et al 2004). Participants self-reported their race as Caucasian, African-American, or other, and the Caucasian sample was further divided into Hispanic or non-Hispanic (Trivedi et al 2006). In addition, Gratacòs and colleagues (2008) reported that no association was found between antidepressant treatment outcome and the BDNF Val66Met polymorphism in the mood disorder (such as MDD and bipolar disorder) patients of Spanish origin who were treated with a variety of antidepressant medications.

The previous findings mainly reported of the association studies of the BDNF Val66Met polymorphism and antidepressants, such as citalopram, milnacipran, fluvoxamine, and fluoxetine. In this work, our goal was to determine the relationship between the polymorphism Val66Met in the BDNF gene and the short-term antidepressant response to venlafaxine, a serotonin and norepinephrine reuptake inhibitor (SNRI), in a Taiwanese population with MDD.

\section{Materials and methods}

\section{Patients}

Blood samples were collected from 72 Taiwanese patients with MDD during the years 2006-2008. All patients were enrolled from the Chi Mei Medical Center Liouying Branch, National Cheng Kung University Hospital, and National Taiwan University Hospital Yun-Lin Branch in Taiwan. Eligibility was limited to patients with a diagnosis of MDD according to the Diagnostic and Statistical Manual of Mental
Disorders IV criteria and the Hamilton Rating Scale for Depression (HAM-D) scores $\geq 15$. Other inclusion criterion was age $\geq 18$ years. Exclusion criteria for all subjects included (1) another major psychiatric illness; (2) active alcohol or drug dependence; (3) primary neurological illness, including dementia. This study was approved by the Ethics Committees of the Chi Mei Medical Center, National Cheng Kung University Hospital, and National Taiwan University Hospital, and conformed to the principles of the Declaration of Helsinki. Informed consent was obtained from each patient.

All of the patients had received venlafaxine antidepressant therapy for 4 weeks. Daily doses of venlafaxine were given, starting at $75 \mathrm{mg} /$ day. Physicians could increase the dosage to $150 \mathrm{mg} /$ day based on the clinical response. Patients that showed a minimum 50\% decrease in the HAM-D score after 4 weeks of treatment were defined as rapid responders to this treatment.

\section{Genotyping}

Genomic DNAs were extracted from each of the blood samples by using QIAamp DNA Blood kit according to the manufacture's instructions. The quality of the extracted genomic DNAs was checked by agarose gel electrophoresis analysis and stored at $-80{ }^{\circ} \mathrm{C}$ until use.

Genomic DNA (15-40 ng) was amplified using a commercially available AmSure SNP detection kit (Vita Genomics, Inc., Taiwan) according to the manufacture's instruction. Amplification conditions consisted of a 10 minutes pre-incubation at $95^{\circ} \mathrm{C}$ to activate the Taq DNA polymerase, followed by 50 cycles of denaturation at $95^{\circ} \mathrm{C}$ for 15 seconds and then primer annealing and extension for 1 minute at $60{ }^{\circ} \mathrm{C}$. After amplification, the fluorescence intensity of the PCR product was measured from $60{ }^{\circ} \mathrm{C}$ to $95^{\circ} \mathrm{C}$ at a temperature gradient of $0.4^{\circ} \mathrm{C} / 15$ seconds. Amplification and dissociation were carried out using 7900HT Fast Real-Time PCR System (ABI, USA), and the ABI 7900 automatically calculated the negative derivative of the change in fluorescence. The SNP genotype of each tested sample was determined by computer software and was confirmed manually.

\section{Statistical analysis}

The $\chi^{2}$ test was used to analyze the categorical data. Furthermore, we compared differences for continuous variables using the Student's t-test. In addition, genotype frequencies were evaluated for Hardy-Weinberg equilibrium using a $\chi^{2}$ goodness-of-fit test. All statistical tests were two-tailed, and p-values less than 0.05 were 
considered statistically significant. Data are presented as mean \pm standard deviation.

\section{Results}

As described previously, we collected 72 samples in MDD patients who have been treated with venlafaxine, including 38 rapid responders and 34 nonresponders. The SNP genetic markers of these subjects were generated at the high-throughput genomics lab of Vita Genomics, Inc. Table 1 demonstrates the demographic characteristics of study subjects. On average, $52.8 \%$ of patients were rapid responders. There was no strong statistical relationship between age and short-term responsiveness as well as between gender and short-term responsiveness. The age and gender distributions of rapid responder and nonresponders were similar ( $p$-value $=0.72$ and $p$-value $=0.11$, respectively). In addition, genotype frequency distributions for the BDNF Val66Met polymorphism were in Hardy-Weinberg equilibrium.

Table 2 shows short-term antidepressant response for the BDNF Val66Met genotypes for the MDD patients. Overall, the BDNF Val66Met polymorphism was assessed and was found not to be associated with short-term venlafaxine treatment outcome (Table 2). In addition, a genderspecific association was identified, such that the BDNF Val66Met polymorphism showed a trend $(\mathrm{p}$-value $=0.077)$ to be associated with short-term venlafaxine treatment response in female patients. However, the BDNF Val66Met polymorphism showed no association with short-term venlafaxine treatment response in male patients.

\section{Discussion}

To the best of our knowledge, this is the first study that proposes to investigate whether the Val66Met polymorphism in the BNDF gene is related to a 4-week venlafaxine antidepressant effect in Taiwanese MDD patients. Evidence for relevance of BDNF to antidepressant treatment outcome is currently inconsistent. In Korean (Choi et al 2006) and Japanese (Yoshida et al 2007) samples, the BNDF Val66Met polymorphism was found to be associated with antidepressant efficacy. Conversely, no association was found between antidepressant treatment outcome and the BNDF Val66Met polymorphism in Caucasian, African-American (McMahon et al 2006; Paddock et al 2007), and Spanish (Gratacòs et al 2008) cohorts. Furthermore, our findings were similar to another study of Taiwanese patients (Tsai et al 2003), which reported no link but a trend $(\mathrm{p}$-value $=0.086)$ to improved 4-week-fluoxetine antidepressant response in heterozygous carriers. The potential reasons for the discrepancies among these studies may be the sample sizes, different ethnicities, different study designs, different definitions of responders, or different antidepressants. The studies that were conducted on small patient populations may also have biased a particular result. Future research with large sample sizes is needed to confirm the findings identified in this study.

Some studies (Monteggia et al 2004; Chen et al 2006) employed mice with impaired BDNF signaling to investigate behavior following antidepressant treatments and indicated that impairment of BDNF signaling may lead to a blunted behavioral response to antidepressants. Therefore, these studies suggest that BDNF plays an important role in the action of antidepressants (Wang et al 2008). It is interesting to point out that although Gratacòs and colleagues (2008) did not find a significant involvement of the BNDF Val66Met polymorphism in treatment outcome, they found evidence for a significant association between remission and the SNP rs908867 located in the BDNF 5' upstream region.

In this work, we identified gender-specific SNP/gene contributions to antidepressant treatment response reporting that the BDNF Val66Met polymorphism showed a trend to be associated with short-term venlafaxine treatment response in female patients. In contrast, no significant difference was found in male patients. Some findings are consistent with the hypothesis that major depression may be related to distinct genes in male versus female patients (Kendler et al 2006; Lin and Hsu 2008). A recent study demonstrated that there were gender differences in BDNF conditional knockout

Table I Demographic information of study subjects. Data are presented as mean \pm standard deviation

\begin{tabular}{lll}
\hline Factor & Rapid responder & Nonresponder \\
\hline $\mathrm{n}(\%)$ & $38(52.8 \%)$ & $34(47.2 \%)$ \\
HAM-D score at baseline & $27.1 \pm 6.7$ & $29.4 \pm 5.7$ \\
HAM-D score at week 4 & $8.3 \pm 4.2$ & $20.2 \pm 5.0$ \\
Age & $43.8 \pm 13.9$ & $42.7 \pm 11.1$ \\
Male/Female, $\mathrm{n}$ & $10 / 28$ & $15 / 19$ \\
\hline
\end{tabular}

Abbreviation: HAM-D, hamilton rating scale for depression. 
Table 2 Short-term antidepressant response for the BDNF genotypes for major depressive disorders

\begin{tabular}{|c|c|c|c|c|}
\hline \multirow[t]{2}{*}{ Factor } & \multicolumn{3}{|c|}{ BDNF genotype } & \multirow[t]{2}{*}{ p-value } \\
\hline & Val/Val & Val/Met & Met/Met & \\
\hline Rapid responder vs nonresponder & $8 / 6$ & $20 / 21$ & $9 / 7$ & 0.7155 \\
\hline \multicolumn{5}{|l|}{ Male } \\
\hline Rapid responder vs nonresponder & $1 / 5$ & $5 / 8$ & $4 / 2$ & 0.1262 \\
\hline \multicolumn{5}{|l|}{ Female } \\
\hline Rapid responder vs nonresponder & $7 / 1$ & $16 / 13$ & $5 / 5$ & 0.0772 \\
\hline Gender (male/female) & $6 / 8$ & $13 / 29$ & $6 / 10$ & 0.4763 \\
\hline
\end{tabular}

Abbreviation: BDNF, brain-derived neurotrophic factor.

mice (Monteggia et al 2007). Treatment response may therefore follow a similar division between men and women, and recent data indicate that the efficacy of selective serotonin reuptake inhibitors (SSRIs) may be influenced by gender differences (Khan et al 2005; Pinto-Meza et al 2006; Berlanga et al 2006; Naito et al 2007).

Furthermore, some studies revealed gender-specific associations between antidepressant treatment response and the following candidate genes: catechol-O-methyltransferase (COMT); glutamate receptor, ionotropic, kainate 1 (GRIK1); glutamate receptor, ionotropic, N-methyl D-aspartate 2A (GRIN2A); 5-hydroxytryptamine receptor 1A (HTR1A; serotonin receptor 1A); monoamine oxidase A (MAOA). From the STAR*D cohort, they reported that the GRIN2A gene and the GRIK1 gene showed significant association with treatment response in female patients treated with citalopram (Paddock et al 2007). Moreover, a study of Taiwanese MDD patients investigated the polymorphism C-1019G of the HTR1A gene with a 4-week fluoxetine antidepressant effect and identified that female patients with the $-1019 \mathrm{C} / \mathrm{C}$ genotype showed a better response than $-1019 \mathrm{G}$ carriers (Yu et al 2006). Also, in another study of MDD patients of Caucasian descent who were treated with a variety of antidepressant medications, poor outcome in the female subjects showed association with the COMT $158 \mathrm{Val} / \mathrm{Val}$ genotype (Baune et al 2008). In addition, it was suggested that female mirtazapine-treated patients homozygous for the T-allele of the MAOA T941G polymorphism had a significantly faster and better treatment response than TG/GG-patients in a study of German MDD patients (Tadić et al 2007). Similarly, another study of a Taiwanese population reported that female MDD patients with 3-repeat homozygotes in the promoter region of the MAOA gene had a significantly better response to 4-week fluoxetine treatment when compared with 4-repeat allele carriers (Yu et al 2005).

In routine clinical practice, a patient would likely switch the mediation or at a second drug at latest after 2 or 3 weeks of unsuccessful antidepressant treatment (Kirchheiner et al 2007). Therefore, we were particularly interested in the rapid response to antidepressant medications after 4 weeks. Furthermore, previous studies have found that there was an excellent correlation between early response and final outcome (Binder et al 2004; Szegedi et al 2005). In this study, $52.8 \%$ of participants met the response criterion already after a 4-week treatment. Other studies also suggested associations between short-term antidepressant treatment response and the following candidate genes: angiotensin II receptor-associated protein (AGTRAP), 5-hydroxytryptamine receptor 2A (HTR2A; serotonin receptor 2A), solute carrier family 6 member 3 (SLC6A3; dopamine neurotransmitter transporter), and solute carrier family 6 member 4 (SLC6A4; serotonin neurotransmitter transporter). In a Korean population of MDD patients treated with mirtazapine, Kang and colleagues (2007) genotyped the serotonin transporter gene-linked polymorphic region (5-HTTLPR) polymorphism in the SLC6A4 gene and reported that responses at the 2 nd and 4 th weeks were significantly better for the short/short genotype of the 5-HTTLPR polymorphism than for long-allele carriers. Similarly, based on a sample of Japanese patients with MDD, paroxetine was found to be significantly more effective than fluvoxamine in the short/short carriers of the SLC6A4 5-HTTLPR polymorphism after 4 weeks of medication (Kato et al 2005). Moreover, Kirchheiner and colleagues (2007) demonstrated that a 40-basepair variable number of tandem repeats in the SLC6A3 gene influenced rapid response to various antidepressants after 3-week therapy in a sample of Caucasian MDD patients. Also, it was found that there were significant differences in short-term antidepressant response according to the $-1438 \mathrm{~A} / \mathrm{G}$ variation of the HTR2A gene in a sample of Korean MDD patients treated with citalopram for 4 weeks (Choi et al 2005). Finally, it was revealed that the A1166C polymorphism of the AGTRAP 
gene was associated with poorer improvement in HAM-D scale after 4 weeks of treatment with various antidepressants (Bondy et al 2005).

There were several limitations to this study as follows. First, in the absence of a placebo control, it could not be determined whether the observed associations were directly attributable to antidepressant treatment. In this outpatient population of the present study, another issue was the lack of a standard measure of adherence (that is, serum level monitoring), which could also have affected the results. Moreover, the definitions of response and remission may affect the findings. Finally, because participants in this study were treated only with venlafaxine, these findings may not be generalizable to other antidepressant medications.

In future work, the contributions of other markers in the BDNF gene should be further examined. Moreover, a previous study supports the implication of the solute carrier family 6 member 2 (SLC6A2; norepinephrine neurotransmitter transporter) gene in SNRI antidepressant treatment outcome in MDD (Yoshida et al 2004). In future work, we aim to investigate the relationship between the SLC6A2 gene and the antidepressant response to venlafaxine (an SNRI) in a Taiwanese population with MDD. In addition, future research using pattern recognition approaches (Lin et al 2006b; Lin and Huang 2008; Lin and Hwang 2008) is needed in order to model associations between gene variants and antidepressant response, as well as to evaluate gene-gene and gene-environment interactions (Lin et al 2007a, 2007b) among genes and clinical factors.

\section{Conclusion}

In this study, we proposed to determine the relationship between the Val66Met polymorphism in the BDNF gene and the rapid antidepressant response to venlafaxine in a Taiwanese population with MDD. Overall, the BDNF Val66Met polymorphism was found not to be associated with short-term venlafaxine treatment outcome. An interesting finding was that the BDNF Val66Met polymorphism showed a trend to be associated with short-term venlafaxine treatment response in female patients. Independent replications in large sample sizes are needed to confirm the role of the BDNF Val66Met polymorphism found in this study in antidepressant treatment response.

\section{Disclosure}

The authors report no conflicts of interest in this work.

\section{Acknowledgments}

The authors extend their sincere thanks to Vita Genomics, Inc. for funding this research. This work was partially supported by the Department of Health, Taiwan (DOH96TD-D-113-041). The authors would also like to thank the anonymous reviewers for their constructive comments, which improved the context and the presentation of this paper.

\section{References}

Bath KG, Lee FS. 2006. Variant BDNF (Val66Met) impact on brain structure and function. Cogn Affect Behav Neurosci, 6:79-85.

Baune BT, Hohoff C, Berger K, et al. 2008. Association of the COMT val158met Variant with Antidepressant Treatment Response in Major Depression. Neuropsychopharmacology, 33:924-32.

Berlanga C, Flores-Ramos M. 2006. Different gender response to serotonergic and noradrenergic antidepressants: A comparative study of the efficacy of citalopram and reboxetine. J Affect Disord, 95(1-3):119-23.

Binder EB, Salyakina D, Lichtner P, et al. 2004. Polymorphisms in FKBP5 are associated with increased recurrence of depressive episodes and rapid response to antidepressant treatment. Nat Genet, 36:1319-25.

Bondy B, Baghai TC, Zill P, et al. 2005. Genetic variants in the angiotensin I-converting-enzyme (ACE) and angiotensin II receptor (AT1) gene and clinical outcome in depression. Prog Neuropsychopharmacol Biol Psychiatry, 29:1094-9.

Chen ZY, Jing D, Bath KG, et al. 2006. Genetic variant BDNF (Val66Met) polymorphism alters anxiety-related behavior. Science, 314:140-3.

Choi MJ, Kang RH, Lim SW, et al. 2006. Brain-derived neurotrophic factor gene polymorphism (Val66Met) and citalopram response in major depressive disorder. Brain Res, 1118:176-82.

Choi MJ, Kang RH, Ham BJ, et al. 2005. Serotonin receptor 2A gene polymorphism $(-1438 \mathrm{~A} / \mathrm{G})$ and short-term treatment response to citalopram. Neuropsychobiology, 52:155-62.

Gratacòs M, Soria V, Urretavizcaya M, et al. 2008. A brain-derived neurotrophic factor (BDNF) haplotype is associated with antidepressant treatment outcome in mood disorders. Pharmacogenomics J, 8:101-12.

Groves JO. 2007. Is it time to reassess the BDNF hypothesis of depression? Mol Psychiatry, 12:1079-88.

Hwang JP, Tsai SJ, Hong CJ, et al. 2006. The Val66Met polymorphism of the brain-derived neurotrophic-factor gene is associated with geriatric depression. Neurobiol Aging, 27:1834-7.

Kang RH, Wong ML, Choi MJ, et al. 2007. Association study of the serotonin transporter promoter polymorphism and mirtazapine antidepressant response in major depressive disorder. Prog Neuropsychopharmacol Biol Psychiatry, 31:1317-21.

Kato M, Ikenaga Y, Wakeno M, et al. 2005. Controlled clinical comparison of paroxetine and fluvoxamine considering the serotonin transporter promoter polymorphism. Int Clin Psychopharmacol, 20:151-6.

Kendler KS, Gatz M, Gardner CO, et al. 2006. A Swedish national twin study of lifetime major depression. Am J Psychiatry, 163:109-14.

Kessler RC, Berglund P, Demler O, et al. 2003. National Comorbidity Survey Replication. The epidemiology of major depressive disorder: results from the National Comorbidity Survey Replication (NCS-R). JAMA, 289:3095-105.

Khan A, Brodhead AE, Schwartz KA, et al. 2005. Sex differences in antidepressant response in recent antidepressant clinical trials. J Clin Psychopharmacol, 25:318-24.

Kirchheiner J, Nickchen K, Bauer M, et al. 2004. Pharmacogenetics of antidepressants and antipsychotics: the contribution of allelic variations to the phenotype of drug response. Mol Psychiatry, 9:442-73.

Kirchheiner J, Nickchen K, Sasse J, et al. 2007. A 40-basepair VNTR polymorphism in the dopamine transporter (DAT1) gene and the rapid response to antidepressant treatment. Pharmacogenomics $J$, $7: 48-55$. 
Lin E, Hwang Y, Tzeng CM. 2006a. A case study of the utility of the HapMap database for pharmacogenomic haplotype analysis in the Taiwanese population. Mol Diagn Ther, 10:367-70.

Lin E, Hwang Y, Wang SC, et al. 2006b. An artificial neural network approach to the drug efficacy of interferon treatments. Pharmacogenomics, 7:1017-24.

Lin E, Hwang Y, Liang KH, et al. 2007a. Pattern-recognition techniques with haplotype analysis in pharmacogenomics. Pharmacogenomics, 8:75-83.

Lin E, Hwang Y, Chen EY. 2007b. Gene-gene and gene-environment interactions in interferon therapy for chronic hepatitis C. Pharmacogenomics, 8:1327-35.

Lin E, Chen PS. 2008. Pharmacogenomics with antidepressants in the STAR*D study. Pharmacogenomics, 9:935-46.

Lin E, Hsu SY. 2008. Gender differences and pharmacogenomics with antidepressants in depression. In: Hernandez $\mathrm{P}$ and Alonso S (eds). Women and Depression. New York: Nova Science Publishers.

Lin E, Huang LC. 2008. Identification of significant genes in genomics using Bayesian variable selection methods. Computational Biology and Chemistry: Advances and Applications, 1:13-8.

Lin E, Hwang Y. 2008. A support vector machine approach to assess drug efficacy of interferon and ribavirin combination therapy. Mol Diagn Ther, 12:219-23.

McMahon FJ, Buervenich S, Charney D, et al. 2006. Variation in the gene encoding the serotonin $2 \mathrm{~A}$ receptor is associated with outcome of antidepressant treatment. Am J Hum Genet, 78:804-14.

Monteggia LM, Barrot M, Powell CM, et al. 2004. Essential role of brain-derived neurotrophic factor in adult hippocampal function. Proc Nat Acad Sci US A, 101:10827-32.

Monteggia LM, Luikart B, Barrot M, et al. 2007. Brain-derived neurotrophic factor conditional knockouts show gender differences in depressionrelated behaviors. Biol Psychiatry, 61:187-97.

Naito S, Sato K, Yoshida K, et al. 2007. Gender differences in the clinical effects of fluvoxamine and milnacipran in Japanese major depressive patients. Psychiatry Clin Neurosci, 61:421-7.

Paddock S, Laje G, Charney D, et al. 2007. Association of GRIK4 with outcome of antidepressant treatment in the STAR*D cohort. Am J Psychiatry, 164:1181-8.

Pinto-Meza A, Usall J, Serrano-Blanco A, et al. 2006. Gender differences in response to antidepressant treatment prescribed in primary care. Does menopause make a difference? J Affect Disord, 93(1-3):53-60.
Ribeiro L, Busnello JV, Cantor RM, et al. 2007. The brain-derived neurotrophic factor rs6265 (Val66Met) polymorphism and depression in Mexican-Americans. Neuroreport, 18:1291-3.

Rush AJ, Fava M, Wisniewski SR, et al. 2004. Sequenced treatment alternatives to relieve depression (STAR*D): rationale and design. Control Clin Trials, 25:119-42.

Serretti A, Artioli P. 2004. The pharmacogenomics of selective serotonin reuptake inhibitors. Pharmacogenomics $J, 4: 233-44$.

Szegedi A, Rujescu D, Tadic A, et al. 2005. The catechol-O-methyltransferase Val108/158Met polymorphism affects short-term treatment response to mirtazapine, but not to paroxetine in major depression. Pharmacogenomics J, 5:49-53.

Tadić A, Müller MJ, Rujescu D, et al. 2007. The MAOA T941G polymorphism and short-term treatment response to mirtazapine and paroxetine in major depression. Am J Med Genet B Neuropsychiatr Genet, 144:325-31.

Trivedi MH, Rush AJ, Wisniewski SR, et al. 2006. Evaluation of outcomes with citalopram for depression using measurement-based care in STAR*D: implications for clinical practice. Am J Psychiatry, $163: 28-40$

Tsai SJ, Cheng CY, Yu YW, et al. 2003. Association study of a brain-derived neurotrophic-factor genetic polymorphism and major depressive disorders, symptomatology, and antidepressant response. Am J Med Genet B Neuropsychiatr Genet, 123:19-22.

Wang JW, Dranovsky A, Hen R. 2008. The when and where of BDNF and the antidepressant response. Biol Psychiatry, 63:640-1.

Yoshida K, Takahashi H, Higuchi H, et al. 2004. Prediction of antidepressant response to milnacipran by norepinephrine transporter gene polymorphisms. Am J Psychiatry, 161:1575-80.

Yoshida K, Higuchi H, Kamata M, et al. 2007. The G196A polymorphism of the brain-derived neurotrophic factor gene and the antidepressant effect of milnacipran and fluvoxamine. J Psychopharmacol, 21:650-6.

Yu WY, Tsai SJ, Hong CJ, et al. 2005. Association study of a monoamine oxidase A gene promoter polymorphism with major depressive disorder and antidepressant response. Neuropsychopharmacology, 30:1719-23.

Yu YW, Tsai SJ, Liou YJ, et al. 2006. Association study of two serotonin $1 \mathrm{~A}$ receptor gene polymorphisms and fluoxetine treatment response in Chinese major depressive disorders. Eur Neuropsychopharmacol, 16:498-503. 